\title{
ORIGINAL
}

\section{VALIDACIÓN DEL MODELO REI PARA MEDIR LA CLASE SOCIAL EN POBLACIÓN ADULTA (*)}

\author{
Antonio Cabrera de León (1, 2), María C Rodríguez Pérez (1), Santiago Domínguez Coello (1), \\ Concepción Rodríguez Díaz (1), Cristobalina Rodríguez Álvarez (2), Armando Aguirre Jaime (1) \\ y el grupo CDC**
}

(1) Unidad de Investigación de la Gerencia de Atención Primaria y del Hospital Universitario Nuestra Señora de la Candelaria. Santa Cruz de Tenerife.

(2) Área de Medidina Preventiva. Universidad de La Laguna.

(3) Servicio de Medicina Interna. Hospital Dr. Negrín. Gran Canaria.

\section{RESUMEN}

Fundamento: La clase social generalmente se mide de manera categórica y basada en la ocupación laboral, lo cual tiene múltiples limitaciones. El objetivo de este trabajo es elaborar un indicador cuantitativo de clase social, fácilmente estandarizable, validarlo en población adulta y comprobar su aptitud para medir el impacto de la clase social como determinante de salud.

Métodos: estudio transversal de 6.729 individuos para medir la clase social con las variables: Renta familiar per cápita, Indice de hacinamiento, Estudios realizados, Ocupación laboral y Situación laboral. Se crearon dos modelos y mediante curvas COR se seleccionó el mejor para validarlo analizando su capacidad de estimar los riesgos relativos de: residir en barrio pobre o rico, mantener un patrón dietético típico de clases sociales pobres y presentar problemas de salud actualmente asociados a la pobreza.

Resultados: el modelo sólo incluyó las variables Renta, Estudios e Índice de hacinamiento (REI), produjo un indicador con rango de valores entre 4 y 21 y mostró correlación inversa con la edad $(r=-0,28 ; p<0,001)$, con el consumo de papas $(r=-0,17 ; p<0,001)$ y con el consumo de legumbres $(\mathrm{r}=-0,03 ; \mathrm{p}=0,01)$, además de correlación directa con el consumo de ensalada $(r=0,10 ; p<0,001)$; su sensibilidad para detectar la residencia en barrio pobre alcanzó el $97 \%$ para valores menores a 10. REI estimó que las clases sociales pobres presentan riesgos significativos de situación laboral de desempleo $(\mathrm{OR}=5,4)$, ocupación laboral de baja cualificación $(\mathrm{OR}=40,9)$, habitar en barrios pobres $(\mathrm{OR}=30,2)$, bajo consumo de ensaladas $(\mathrm{OR}=2,2)$, gran consumo de papas $(\mathrm{OR}=4,5)$ y alto consumo de legumbres $(\mathrm{OR}=1,6)$. En ambos sexos las clases pobres presentaron mayor riesgo de problemas de salud, con mayor fuerza en las mujeres: sedentarismo ( $\mathrm{OR}=1,8)$, obesidad $(\mathrm{OR}=4,4)$, obesidad abdominal (OR $=5,4)$, síndrome metabólico $(\mathrm{OR}=3,4)$ y diabetes mellitus $(\mathrm{OR}=2,0)$.

Conclusiones: REI es un indicador válido, no basado en la ocupación ni en la situación laboral, fácilmente estandarizable, apto para medir cuantitativamente la clase social en estudios que precisen analizar el impacto de la misma como determinante de salud.

Palabras clave: Clase social. Pobreza.

\section{Correspondencia:}

Dr. Antonio Cabrera de León

Hospital San Juan de Dios.

Ctra. Santa Cruz - La Laguna, 53.

38009. Santa Cruz de Tenerife

Correo electrónico: acabrera@sjd.es
ABSTRACT

\section{Validation of the ICE Model to Assess Social Class in the Adult Population}

Background: Social class has commonly been defined by the type of employment and it is assessed as a categorical variable. However, this approach has a number of drawbacks. The objective of this article is to develop and validate a readily standardizable quantitative indicator of social class and to show its ability to measure the impact of social class as a health determinant.

Methods: In 6729 individuals we measured income, crowding index, education, occupation and employment status. Two models were adjusted to study the neighborhood, dietary pattern and health problems.

Results: The model that included only income, crowding index and education (ICE) yielded an indicator that correlated with age $(\mathrm{r}=$ $-0.28 ; \mathrm{p}<0.001)$ and consumption of potatoes $(\mathrm{r}=-0.17 ; \mathrm{p}<0.001)$ and salads $(r=0.10 ; p<0.001)$. This indicator estimated that poor social classes were at significant risk for unemployment $(\mathrm{OR}=5$,$) , blue$ collar jobs $(\mathrm{OR}=40,9)$, residing in poor neighborhoods $(\mathrm{OR}=30.2)$, low salad consumption $(\mathrm{OR}=2.2)$ and high consumption of potatoes $(\mathrm{OR}=4.5)$. They also had, especially in women, a higher risk of sedentarism $(\mathrm{OR}=1.8)$, obesity $(\mathrm{OR}=4.4)$, metabolic syndrome (OR $=3.4)$ and diabetes mellitus $(\mathrm{OR}=2.0)$.

Conclusions: The ICE index was valid, not based on occupation or employment status, readily standardizable, and suitable for measuring social class and its impact of on health.

Keywords: Social class. Poverty.

\footnotetext{
* Financiado por la Fundación Canaria de Investigación y Salud y por el Fondo de Investigación Sanitaria (PI 070934) ** Grupo CDC: Basilio Anía Lafuente (3), Francisco Hernández Díaz (1), Carlos Borges Álamo (1), Buenaventura Brito Díaz (1), Delia Almeida González (1), Ana González Hernández (1), Lourdes Carrillo Fernández (1), José Carlos del Castillo Rodríguez (1), Noelia Fernández Ramos (3), y José Juan Alemán Sánchez (1).
} 


\section{INTRODUCCIÓN}

La Organización Mundial de la Salud considera que la pobreza es la principal causa de enfermedades. Las desigualdades socioeconómicas son los factores que explican mejor las diferencias de salud dentro de una misma sociedad ${ }^{1-3}$. Los grupos sociales más ricos y educados adquieren antes hábitos de vida saludables, de manera que en ellos disminuyen los factores de riesgo y las enfermedades, mientras que éstas se mantienen elevadas en las capas sociales desfavorecidas ${ }^{4}$.

Se mida en el país que se mida, el estado de salud y las tasas de mortalidad son mucho peores en las clases sociales pobres, aunque la magnitud de las diferencias entre pobres y ricos depende de la distribución de la riqueza en cada comunidad y de las oportunidades para la educación que en la misma se den 5 . Tradicionalmente, las clases sociales han sido definidas por el tipo de trabajo que se ejerce y por ello la mayoría de los autores continúa midiendo la clase social categóricamente a través de la ocupación laboral, bien sola ${ }^{6,7}$ o bien combinada con otras variables como la educación y los ingresos económicos ${ }^{2,8}$. Pero esto tiene numerosos inconvenientes: el primero de ellos es que la clasificación e importancia social de las ocupaciones laborales cambia con el tiempo y es difícil estandarizar la clase social para comparación de momentos distintos incluso en un mismo país. Además, la ocupación laboral se clasifica de manera diferente en muchos países, lo cual es un obstáculo más para estandarizarla y comparar comunidades distintas. En España se ha propuesto una clasificación para medir la clase social ${ }^{9}$, inspirada en la propuesta previa de Goldthorpe ${ }^{10}$. Esta medición, basada en la ocupación laboral, resulta igualmente difícil de estandarizar, de hecho el mismo autor la modifica en función de los objetivos estudiados ${ }^{11}$. Otro inconveniente es que no permite clasificar a los individuos que declaran ciertas situacio- nes laborales (faenas del hogar, estudiantes, parados, jubilados, discapacitados, etc). La situación laboral precaria, particularmente el desempleo, implica falta de ingresos y recursos materiales y refleja por tanto la privación material y social ${ }^{12}$.

La educación, o más bien su escasez, es un indicador de privación material y social $^{12}$ cuyo empleo en la medición de la clase social sigue siendo de máxima importancia pero es omitido por las clasificaciones basadas únicamente en la ocupación. La educación y la renta disponible son componentes principales de la clase social, y la falta de de ambos se asocia a mayor frecuencia de problemas de salud ${ }^{13}$. Otra variable asociada a las clases sociales bajas es el hacinamiento, cuya presencia se asocia a pobreza, mayor demanda de asistencia médica ${ }^{13}$, residencia en áreas con alta polución $^{14}$ o escaso desarrollo antropométrico en la infancia ${ }^{15}$. Algunos autores la incluyen como componente principal de la definición de clase social ${ }^{16}$.

En definitiva, la clase social puede medirse de manera diversa a través de sus múltiples componentes, pero la medición viene realizándose de forma categórica desde sus orígenes puesto que ello está implícito en el propio concepto de clase. Ha habido dos grandes escuelas, marxista y weberiana, que han aportado distinta visión de la misma, pero siempre centrada en el trabajo ${ }^{17}$. Cabe plantear que, con el desarrollo de las clases medias, los límites entre clases sociales se difuminan y resulta conveniente disponer de mediciones cuantitativas de las mismas.

El objetivo de este trabajo es elaborar un sencillo indicador cuantitativo de clase social, cuyos componentes permitan estandarizarlo para comparar comunidades diferentes, validarlo en una amplia muestra de población general adulta y comprobar que es apto para medir el impacto de la clase social como determinante de salud. 


\section{SUJETOS Y MÉTODOS}

Se realizó un estudio transversal con los sujetos participantes en la cohorte denominada "CDC de Canarias" (CDC es el acrónimo de Cardiovascular, Diabetes y Cáncer), que habían sido seleccionados de la población general entre los años 2000 y 2005. La metodología seguida ha sido descrita anteriormente ${ }^{18}$. Se definió sedentarismo como no realizar al menos 30 minutos diarios de actividad física moderada ${ }^{19} \mathrm{y}$ obesidad como padecer un índice de masa corporal $($ IMC) $>30$. Se empleó también el concepto de obesidad abdominal en función de la ratio cintura abdominal/estatura ${ }^{20}$. Para el síndrome metabólico se empleó tanto la definición propuesta por el ATP III $^{21}$ como la sugerida por la $\mathrm{IDF}^{22}$. La diabetes mellitus se definió con los criterios de la $\mathrm{ADA}^{23}$.

Las variables utilizadas para medir la clase social fueron las siguientes:

A) Renta familiar per cápita (cociente entre el promedio de ingresos mensuales obtenidos por todos los miembros de la familia y el número de miembros de la misma). Se empleó categorizado en quintiles.

B) Índice de hacinamiento (cociente entre el número de personas que habita el hogar y el número de dormitorios disponibles). Se le asignó luego un valor de 0 puntos si el resultado del cociente era mayor de 2,1 punto para cocientes de 1 a 2 y 2 puntos si el cociente era menor de 1 .

C) Estudios realizados, medidos en una escala ordinal que refleja los años de educación recibida: 1 punto para las personas analfabetas, 2 puntos para estudios primarios completos o incompletos, 3 puntos para estudios secundarios completos o incompletos y 4 puntos para estudios universitarios completos o incompletos.
D) Ocupación laboral, referida al sector laboral en el que el participante desarrolla su actividad económica. Esta variable se incluyó en el estudio porque, pese a sus desventajas anteriormente comentadas, ha sido históricamente la más empleada para medir la clase social y se tenía el propósito de analizar un modelo de clase que contara con ella frente otro que la excluyera. El cuestionario CDC recoge la Clasificación Nacional de Ocupaciones $^{24}$, que distribuye a los sujetos en grandes grupos; para su ponderación se otorgó a la agricultura 1 punto, 2 a la construcción, 3 a los oficios manuales como carpinteros o mecánicos, 4 a los dedicados al transporte, turismo, restauración, sus labores u otros y 5 a los sectores sanitario, educativo y similares.

E) Situación laboral en la que se encontraba en el momento de ser enrolado en el estudio. Para su ponderación, a los desempleados se les asignó 0 puntos, 2 a los trabajadores, y 1 al resto de situaciones (jubilados, estudiantes, amas de casa, trabajadores en situación de incapacidad laboral).

Con estas variables se crearon dos modelos matemáticos de medición de la clase social. El primero de ellos, inspirado en la propuestas previas $^{9,25}$, incluyó la actividad laboral. El segundo excluyó las variables laborales. Es decir:

Modelo 1: Renta Familiar per cápita, Ocupación Laboral, Situación Laboral, Indice de Hacinamiento, Estudios realizados.

Modelo 2: Renta Familiar per cápita, Estudios realizados, Índice de Hacinamiento.

Análisis estadístico: Las variables continuas se resumieron mediante su media $\pm \mathrm{DE}$ y las categóricas mediante la frecuencia observada y relativa, o bien se presentan como proporciones con sus inter- 
valos de confianza al $95 \%\left(\mathrm{IC}_{95 \%}\right)$. La correlación entre variables continuas se estimó con el coeficiente de Pearson. Se empleó el análisis de varianza para comprobar la asociación de variables continuas con categóricas y la prueba chi cuadrado de Pearson para estudiar la asociación entre variables categóricas.

Puesto que ha sido repetidamente comprobado que en las sociedades desarrolladas el síndrome metabólico ${ }^{21}$ se asocia a las clases sociales bajas ${ }^{26-28}$, para derivar estos modelos se comenzó comprobando la asociación de cada variable componente con el síndrome metabólico en un $10 \%$ de los individuos estudiados ( $\mathrm{n}=673$ ), de los cuales el $23 \%$ padecía el síndrome (n =155). Dado que todas las variables se asociaron al síndrome, se ajustaron los dos modelos mediante regresión logística multivariante empleando el síndrome metabólico como variable dependiente. El indicador que cada modelo produce se obtuvo mediante la suma de puntos asociados a cada una de sus componentes, asignados según el valor de su coeficiente de regresión redondeado. A continuación, conociendo que residir en un vecindario rico (urbanización de lujo) o pobre (barriada de viviendas sociales) es un indicador aceptado de clase social ${ }^{29,30}$, se utilizó a los participantes cuya residencia se ubicaba en dos barrios que inequívocamente respondían a estas características $(n=369)$ para realizar un análisis de curvas características operador-receptor de tipo II (COR), con la residencia como variable de estado, comparándose el estadístico $\mathrm{C}$ o área bajo la curva generada por los dos indicadores de clase social.

El indicador con mejores resultados (modelo 2) fue el sometido a validación en este artículo comprobando su capacidad de estimar en la muestra restante de sujetos estudiados $(n=6729-673=6056)$ los riesgos relativos de: (a) situación laboral de desempleo ${ }^{12}$, (b) ocupación laboral de baja cualificación ${ }^{9}$, (c) residir en barrio pobre $^{29,30}$, (d) mantener un patrón dietético típico de clases sociales pobres: consumo elevado de papas (gramos/día) ${ }^{31}$ y legumbres (gramos/día) ${ }^{32}$, conjuntamente con bajo consumo de ensaladas $\left(\right.$ gramos/día) ${ }^{33}$ y (e) presentar problemas de salud actualmente asociados a la pobreza (sedentarismo ${ }^{34}$, obesidad ${ }^{6,35}$, síndrome metabólico ${ }^{26,36}$ y diabetes ${ }^{37}$ ). Los riesgos relativos fueron estimados a través de la razón de ventaja (OR). Se empleó para ello modelos de regresión logística, ajustados por edad y sexo, con la clase social como variable independiente, expresada en quintiles, y donde la variable dependiente fue en cada ocasión: (a) desempleado frente a trabajador activo [para esta variable se tomó sólo la población con edades comprendidas entre $30 \mathrm{y}$ 60 años, $n=5.578]$; (b) ocupación poco cualificada frente a muy cualificada [categorías IVb y V de la $\mathrm{SEE}^{9}$ versus categorías I, II y IIIa, tomando sólo la población con edades comprendidas entre 30 y 60 años]; (c) el consumo de papas: quintil más alto frente al más bajo; (b) el consumo de legumbres: quintil más alto frente al más bajo; (c) el consumo de ensaladas: quintil más alto frente al más bajo; además se ajustaron también modelos logísticos ajustados por edad separadamente para hombres y mujeres con las siguientes variables dependientes: (d) sedentarismo; (e) obesidad; (f) obesidad abdominal; (g) síndrome metabólico ATP III; (h) síndrome metabólico IDF; (i) diabetes mellitus tipo 2.

Los cálculos se realizaron con el paquete estadístico SPSS $^{\odot}$ versión 15 en español.

\section{RESULTADOS}

La población estudiada estuvo formada por 3.816 mujeres y 2.913 varones. La tabla 1 muestra la distribución de las variables 
Tabla 1

Distribución por sexo de las variables* que integran los modelos de clase social (Estudios realizados, Renta familiar per cápita, Índice de hacinamiento, Ocupación laboral y Situación Laboral) aplicados en la población estudiada (n=6729)

\begin{tabular}{|c|c|c|c|}
\hline & Hombres & Mujeres & $\mathbf{p}$ \\
\hline \multicolumn{4}{|l|}{ Estudios realizados } \\
\hline No sabe leer & $37(1)$ & $62(2)$ & \multirow{4}{*}{0,372} \\
\hline Primarios completos o incompletos & $1.657(57)$ & $2.162(57)$ & \\
\hline Secundarios completos o incompletos & $814(28)$ & $1.025(27)$ & \\
\hline Universitarios completos o incompletos & $399(14)$ & $558(15)$ & \\
\hline \multicolumn{4}{|l|}{ Renta familiar per cápita ( $€ /$ mes) } \\
\hline$\leq 240$ & $534(19)$ & $839(23)$ & \multirow{5}{*}{$<0,001$} \\
\hline De 241 a 370 & $579(24)$ & $875(21)$ & \\
\hline De 371 a 470 & $595(23)$ & $821(21)$ & \\
\hline De 471 a 649 & $555(20)$ & $593(16)$ & \\
\hline$\geq 650$ & $522(19)$ & $491(14)$ & \\
\hline \multicolumn{4}{|l|}{ Índice de hacinamiento } \\
\hline Más de 2 personas por dormitorio & $69(2)$ & $115(3)$ & \multirow{3}{*}{0,262} \\
\hline Entre 1 y 2 personas por dormitorio & $1.474(51)$ & $1.941(51)$ & \\
\hline 1 persona por dormitorio & $1.355(47)$ & $1.750(46)$ & \\
\hline \multicolumn{4}{|l|}{ Ocupación laboral } \\
\hline Agricultura & $180(6)$ & $296(8)$ & \multirow{8}{*}{$<0,001$} \\
\hline Construcción y minas & $589(21)$ & $20(1)$ & \\
\hline Carpintería, mecánica, industria & $382(14)$ & $71(2)$ & \\
\hline Sanidad, educación, profesiones liberales & $413(15)$ & $897(25)$ & \\
\hline Transporte y reparto & $231(8)$ & $27(1)$ & \\
\hline Turismo, ocio, bares & $452(16)$ & $793(22)$ & \\
\hline Ama de casa & $2(0)$ & $832(23)$ & \\
\hline Otros & $553(20)$ & $663(18)$ & \\
\hline \multicolumn{4}{|l|}{ Situación Laboral } \\
\hline Trabaja & $2.180(75)$ & $1.783(47)$ & \multirow{7}{*}{$<0,001$} \\
\hline Estudia & $97(3)$ & $180(5)$ & \\
\hline Jubilación & $246(8)$ & $54(1)$ & \\
\hline Incapacidad laboral & $146(5)$ & $111(3)$ & \\
\hline Desempleo & $208(7)$ & $284(8)$ & \\
\hline Ama de casa & $5(0)$ & $1.365(36)$ & \\
\hline Otro & $27(1)$ & $32(1)$ & \\
\hline
\end{tabular}

* Resumidas como frecuencias absolutas observadas y frecuencias relativas (\%).

que fueron empleadas para elaborar los distintos modelos de clase social. En ella se aprecia que existen diferencias significativas entre sexos respecto a los ingresos económicos mensuales, la ocupación y la situación laboral.

Los dos modelos de clase social discriminaron claramente entre residencia en zonas ricas o pobres (figura 1). Dado que el modelo 2 alcanzó la mayor área bajo la curva COR, fue el elegido para su validación. A este modelo se le denominó con el acrónimo REI (Renta familiar per cápita, en quintiles $+3 x$ Estudios + $2 x$ Índice de hacinamiento). Medido como variable cuantitativa continua el modelo REI presentó una media de $13,35 \pm 3,42$ sin diferencias significativas entre sexos, con un rango de valores entre 4 y 21 , y mostró correlación inversa con la edad $(\mathrm{r}=-0,28 ; \mathrm{p}<0,001)$, con el consumo de papas $(\mathrm{r}=-0,17 ; \mathrm{p}<0,001)$ y con el consumo de legumbres $(r=-0,03$; 
Figura 1

Área bajo la curva obtenida con 2 modelos de clase social para predecir la zona de residencia

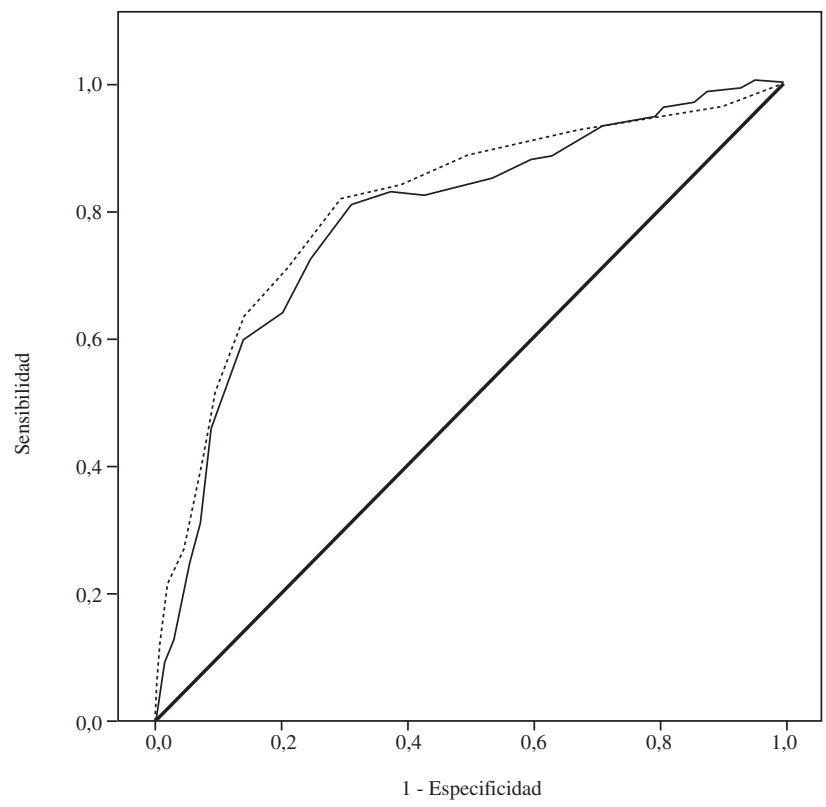

Procedencia de la curva

Clase social: modelo ERIAL

Clase social: modelo RIE

Línea de referencia

Modelo 1: Estudios, Renta, Índice de Hacinamiento, Ocupación Laboral, Situación Laboral.

Modelo $2($ REI $)=$ Estudios, Renta, Índice de Hacinamiento.

\begin{tabular}{|c|c|c|}
\hline Clase social & Área bajo la curva $\left(\mathbf{I C}_{\mathbf{9 5} \%}\right)$ & $\mathrm{p}$ \\
\hline Modelo 1 & $0,787(0,736,0,838)$ & $<0,001$ \\
\hline Modelo 2 (REI) & $0,812(0,763,0861)$ & $<0,001$ \\
\hline
\end{tabular}

$\mathrm{p}=0,01)$, además de correlación directa con el consumo de ensalada $(r=0,10$; $\mathrm{p}=0,001)$. Su sensibilidad para detectar la residencia en barrio pobre alcanzó el $97 \%$ para valores menores a 10 y el $100 \%$ para los menores a 8 .

En la tabla 2 se aprecia que, en análisis bivariados, las clases sociales pobres (quintiles 1 y 2 de REI) presentan mayor edad, mayor desempleo, peor ocupación laboral, consumen menos ensalada, más papas y más legumbres. En general las clases socia- les habitaban en barrios concordantes con su riqueza, aunque hubiera pequeños porcentajes de las clases altas (10\%) y bajas $(5 \%)$ residiendo en el barrio opuesto al que cabría esperar.

El análisis multivariado mostró (tabla 3) que, incluso ajustando el posible efecto de edad y sexo, REI identifica las clases sociales pobres como aquellas que tienen riesgos altos de desempleo, ocupación poco cualificada y un patrón dietético con bajo consumo de ensaladas y gran consu- 
Tabla 2

Distribución de la edad, desempleo, ocupación laboral poco cualificada, ubicación del domicilio en barrio pobre y consumo de algunos alimentos en cada quintil del modelo REI de clase social

\begin{tabular}{|l|c|c|c|c|c|c|}
\hline \multicolumn{1}{|c|}{ Clase Social (REI) } & Quintil 1 & Quintil 2 & Quintil 3 & Quintil 4 & Quintil 5 & p $^{* 1}$ \\
\hline Edad $^{1}$ & $47 \pm 11$ & $47 \pm 13$ & $42 \pm 13$ & $38 \pm 12$ & $38 \pm 11$ & $<0,001$ \\
\hline Situación laboral de desempleo $^{2,3}$ & $10(8,3,11,7)$ & $9(8,5,10,5)$ & $8(6,3,9,7)$ & $6(4,7,7,3)$ & $4(3,4,4,6)$ & $<0,001$ \\
\hline Ocupación laboral de baja cualificación $^{2,3}$ & $93(91,1,94,9)$ & $88(86,5,89,5)$ & $73(69,1,76,9)$ & $56(52,1,59,9)$ & $27(23,4,30,6)$ & $<0,001$ \\
\hline Domicilio en barrio pobre $^{2}$ & $19(14,8,25,2)$ & $32(14,8,25,2)$ & $19(16,6,27,4)$ & $20(18,4,29,6)$ & $10(8,6,17,4)$ & $<0,001$ \\
\hline${\text { Consumo de ensaladas en quintil } \text { bajo }^{2}}^{2}$ & $24(21,7,26,3)$ & $29(26,5,31,5)$ & $17(15,0,19,0)$ & $17(15,0,19,0)$ & $13(11,2,14,8)$ & $<0,001$ \\
\hline Consumo de papas en quintil alto $^{2}$ & $28(25,5,30,5)$ & $31(28,4,33,6)$ & $20(17,7,22,3)$ & $13(11,1,14,9)$ & $8(6,5,9,5)$ & $<0,001$ \\
\hline Consumo de legumbres en quintil alto $^{2}$ & $23(20,2,25,8)$ & $27(24,0,30,0)$ & $20(17,3,22,79)$ & $17(14,5,19,5)$ & $13(10,4,15,6)$ & 0,015 \\
\hline
\end{tabular}

${ }^{1}$ Media \pm DE. ${ }^{2}$ Porcentaje $\left(\mathrm{IC}_{95 \%}\right)$.

* p para la tendencia.

Tabla 3

Estimación de los riesgos relativos de: desempleo, tener una ocupación laboral de baja, residir en zonas pobres y tener determinados consumos de alimentos en cada quintil de clase social. Los riesgos se estimaron mediante regresión logística ajustada por edad y sexo y se expresan como OR $\left(\mathrm{IC}_{95 \%}\right)$

\begin{tabular}{|l|c|c|c|c|c|}
\hline \multicolumn{1}{|c|}{ Clase Social (REI) } & Quintil 1 & Quintil 2 & Quintil 3 & Quintil 4 & Quintil 5 \\
\hline Situación laboral de desempleo & $5,4(3,4,8,6)$ & $4,5(2,9,7,1)$ & $3,0(1,8,5,0)$ & $2,1(1,3,3,5)$ & 1 \\
\hline Ocupación laboral de baja cualificación & $40,9(28,7,58,4)$ & $18,0(13,7,23,7)$ & $7,4(5,8,9,5)$ & $3,4(2,8,4,3)$ & 1 \\
\hline Domicilio en barrio pobre Consumo bajo de ensaladas $^{1}$ & $23,1(8,3,64,5)$ & $30,2(11,9,76,9)$ & $16,1(6,6,39,5)$ & $3,3(1,8,6,4)$ & 1 \\
\hline Consumo alto de papas $^{1}$ & $2,2(1,7,3,0)$ & $2,2(1,7,2,9)$ & $1,8(1,4,2,4)$ & $1,1(0,8,1,4)$ & 1 \\
\hline Consumo alto de legumbres $^{1}$ & $4,5(3,4,6,1)$ & $3,4(2,6,4,6)$ & $3,4(2,5,4,6)$ & $1,6(1,2,2,1)$ & 1 \\
\hline
\end{tabular}

${ }^{1}$ Quintil inferior para consumo de ensaladas y superior para el consumo de papas y legumbres.

Tabla 4

Estimación de los riesgos relativos de padecer sedentarismo, obesidad, síndrome metabólico y diabetes mellitus en el quintil mínimo frente al máximo de clase social según el modelo REI. Los riesgos se estimaron como OR $\left(\mathrm{IC}_{95 \%}\right)$ y se presentan ajustados por edad

\begin{tabular}{|c|c|c|c|c|}
\hline \multirow{2}{*}{ Variable dependiente } & \multicolumn{2}{|c|}{ Hombres } & \multicolumn{2}{|c|}{ Mujeres } \\
\hline & $\mathrm{OR}\left(\mathrm{IC}_{\mathbf{9 5 \%}}\right)$ & $\mathbf{p}$ & OR $\left(\mathrm{IC}_{95 \%}\right)$ & $\mathbf{p}$ \\
\hline Sedentarismo & $1,6(1,2,2,0)$ & $<0,001$ & $1,8(1,4,2,4)$ & $<0,001$ \\
\hline Obesidad & $1,8(1,2,2,4)$ & $<0,001$ & $4,4(3,2,6,1)$ & $<0,001$ \\
\hline Obesidad Abdominal* & $2,2(1,7,2,9)$ & $<0,001$ & $5,4(4,1,7,2)$ & $<0,001$ \\
\hline Síndrome Metabólico ATP & $1,3(1,0,1,8)$ & 0,100 & $3,4(2,4,4,8)$ & $<0,001$ \\
\hline Síndrome Metabólico IDF & $1,4(1,1,1,9)$ & 0,010 & $2,5(1,9,3,4)$ & $<0,001$ \\
\hline Diabetes Mellitus & $1,8(1,1,2,8)$ & 0,016 & $2,0(1,2,3,1)$ & 0,004 \\
\hline
\end{tabular}

* Ratio Cintura abdominal/estatura $>0,55$. ATP $=$ Adult Treatment Panel III. IDF $=$ International Diabetes Federation.

mo de papas. También es mayor en ellas el consumo de legumbres y el riesgo de habitar en barrios pobres.

Ajustando el efecto de la edad, y estratificando el análisis por sexos, el modelo REI identificó las clases pobres como aquellas que presentaban mayor riesgo de padecer problemas de salud, es decir, aquellas que presentaban mayores riesgos de sedentarismo, obesidad, obesidad abdominal, síndrome metabólico y diabetes mellitus (tabla 4). 
El gradiente entre clases pobres y ricas resultó mayor en las mujeres, las cuales presentaron mayores riesgos que los hombres para cada uno de estos problemas de salud.

\section{DISCUSIÓN}

Hemos comprobado la validez del modelo REI para medir la clase social. El mismo produce un indicador sencillo y de fácil obtención mediante entrevista. Un problema tradicional de los modelos de estructura de la sociedad en clases sociales es su falta de validación empírica ${ }^{38}$, a lo cual se añade que los escasos ejemplos de validación se refieren a modelos basados en la ocupación laboral ${ }^{39}$. Comprobada la validez de REI, hay que resaltar que a la sencillez de su obtención añade su capacidad para ser aplicado a cualquier persona sin que, como se comentó anteriormente, haya dificultades para estimarlo en los individuos que declaran ciertas situaciones laborales. Además, al no incluir en su seno la ocupación laboral este indicador cuenta con la ventaja de eludir otro de los problemas arriba descritos para la ocupación: puede ser estandarizado fácilmente $\mathrm{y}$ empleado para comparar comunidades diferentes. Entre los componentes de REI, la renta familiar per cápita recoge una información más real de la riqueza de la que dispone un individuo que la facilitada únicamente por los ingresos personales; la educación es un componente clásico de la clase social puesto que no sólo la riqueza de las personas, sino también sus conocimientos y cultura, definen aquella; por último, la inclusión del índice de hacinamiento en la medición de la clase social matiza la renta disponible y la educación alcanzada con información sobre la forma de vida de los individuos.

No puede considerarse que se disponga de un "patrón oro" de clase social frente al cual validar un constructo teórico que intenta medir la misma, pero son las variables de actividad laboral las más aceptadas para ello. Pues bien, REI muestra riesgos 5 veces mayores de desempleo en las clases pobres, pese a que la información se recogió en los años en que, históricamente, ha habido menos tasa de desempleo en la Comunidad Canaria. Y en cuanto a la ocupación, REI detecta en las clases pobres riesgos superiores a 40 para desempeñar trabajos de baja cualificación. Además, existe un amplio conjunto de variables bien identificadas y aceptadas como indicadores de clase social. En este trabajo se ha optado por el uso, el barrio de residencia, el patrón dietético y algunos problemas de salud típicos de clase social para la validación de REI. En concreto, el lugar de residencia ha sido repetidamente mostrado como marcador de clase tanto dentro ${ }^{40,41}$ como fuera de España $^{29,30,42}$. En nuestro caso, los resultados muestran claramente la validez de REI para encontrar la asociación de cada clase social con el tipo de vecindario donde habita. La detección de OR con valores tan altos es infrecuente en cualquier investigación y habla de la fuerte asociación entre cada clase social y su entorno ambiental previsible. Con la información disponible no es posible saber cuáles son las razones que llevan a un $10 \%$ de la clase rica a habitar en un vecindario pobre. Las complejas redes de relaciones familiares, sociales, económicas, etc, constituyen determinantes que pueden explicar esa variabilidad. Además, el altísimo precio alcanzado por la vivienda en España permite especular con la existencia de capas de población que, pese a que su nivel de educación y de renta les sitúa en la clase alta, residen en barrios más propios de clases bajas.

Respecto al patrón dietético, hemos optado por medirlo a través de alimentos cuyo consumo es sabido que varía con la clase social. En los países ricos, el consumo de papas es hoy claramente más eleva- 
do en las clases pobres ${ }^{31}$. En la población estudiada, adultos de las islas Canarias, esto ha sido también repetidamente confirmado con modelos de clase social basados en la ocupación laboral ${ }^{43,44}$. El modelo REI muestra que un patrón de dieta con un alto consumo de papas es tres veces más frecuente en las clases pobres que en las ricas $(28 \%$ en las pobres versus $8 \%$ en las ricas), lo cual, una vez ajustado por edad y sexo, produce OR superiores a 4 entre ambas clases. Y presenta además una clara tendencia lineal de consumo decreciente conforme aumenta la clase social de la población.

También las legumbres son un grupo de alimentos que ha sido bien identificado por su consumo preferente entre las clases pobres, tanto en Canarias ${ }^{43,44}$ como en otras poblaciones españolas ${ }^{32}$. En este grupo, REI muestra que en las clases pobres el consumo elevado de legumbres es aproximadamente el doble de frecuente que en las ricas. La estimación de riesgos relativos de consumo no produce unos valores tan elevados en este caso como en las papas, pero sí presenta incrementos del $60 \%$ de dichos riesgos en las clases pobres. El hecho de que al aumentar la clase la tendencia del consumo no sea linealmente decreciente puede atribuirse a la supervivencia de la dieta mediterránea y a los consejos dietéticos para mantener una frecuente ingesta de legumbres, lo cual parece ser seguido por buena parte de las clases altas.

Por último, en lo que a alimentos se refiere, el grupo de las ensaladas ha sido repetidamente identificado como de consumo elevado más frecuente entre las clases ricas tanto dentro ${ }^{43,44}$ como fuera de España $^{33}$. Concordando con ello REI identifica en las clases pobres un consumo bajo de ensaladas dos veces más frecuentes que en las ricas, y las OR ajustadas por edad y sexo presentan valores superiores a 2 entre dichas clases. La tendencia lineal del bajo consumo de ensaladas es claramente decreciente al aumentar la clase social.

Hemos analizado problemas de salud de alta prevalencia en las sociedades modernas para los cuales se había comprobado previamente su mayor frecuencia en clases pobres: sedentarismo ${ }^{34}$, obesidad ${ }^{6,35}$, síndrome metabólico ${ }^{26,36}$ y diabetes mellitus ${ }^{37}$. Estratificando por sexos, la aplicación de REI muestra no sólo que son las clases pobres las que sufren mayores riesgos sino que el gradiente entre clases pobres y ricas tiene mayores repercusiones de salud en las mujeres. Este mayor riesgo en las mujeres coincide con lo descrito en poblaciones españolas respecto a la salud percibida ${ }^{45}, \mathrm{y}$ en otras poblaciones se ha comprobado que la estructura social (diferencias socioeconómicas, apoyo familiar, etc) es un determinante más importante para la salud de las mujeres mientras que los determinantes conductuales (alcohol, actividad física, etc) son más importantes para la salud de los hombres ${ }^{46}$.

Como limitación de nuestro estudio se podría argumentar que la selección del índice REI basado en el análisis de áreas bajo la curva empleando una variable de estado (residencia), para después validarlo respecto a la misma variable por la que se selecciona, es un argumento circular. Sin embargo, consideramos que REI queda bien validado con los resultados obtenidos respecto al desempleo, ocupación laboral, patrón dietético, a los problemas de salud analizados y a la distribución por sexo de los mismos. Los resultados respecto a la zona de residencia conviene mostrarlos por cuanto las diferencias obtenidas entre clases son muy importantes y no hacen más que refrendar la validación de REI obtenida frente a los otros marcadores de clase.

En conclusión, REI es un indicador de clase social de fácil elaboración, cuya 
validez hemos comprobado en la población general adulta de Canarias. Al no incluir la ocupación laboral entre sus componentes se puede estandarizar fácilmente y su empleo ofrece una forma sencilla de medir cuantitativamente la clase social en los estudios que precisen analizar el impacto de la misma como determinante de salud.

\section{BIBLIOGRAFÍA}

1. Ranjit N, Diez-Roux AV, Chambless L, Jacobs DR Jr, Nieto FJ, Szklo M. Socioeconomic differences in progression of carotid intima-media thickness in the Atherosclerosis Risk in Communities study. Arterioscler Thromb Vasc Biol. 2006; 26: 411416.

2. Kristensen PL, Wedderkopp N, Andersen LB, Bai $\mathrm{CN}$, Froberg K. Tracking and prevalence of cardiovascular disease risk factors across socioeconomic classes: a longitudinal substudy of the European Youth Heart Study. BMC Public Health. 2006; 6: 20.

3. Gravlee CC, Dressler WW, Bernard HR. Skin color, social classification, and blood pressure in southeastern Puerto Rico. Am J Public Health. 2005; 95: 2191-2197.

4. Monteiro CA, Moura EC, Conde WL, Popkin BM. Socieconomic status and obesity in adult populations of developing countries: a review. Bull World Health Organ 2004; 82: 940-946.

5. Mackenbach JP, Stirbu I, Roskam AJ, Schaap MM, Menvielle G, Leinsalu M, Kunst AE, and the European Union Working Group on Socioeconomic Inequalities in Health. Socioeconomic inequalities in health in 22 European countries. N Engl J Med. 2008; 358: 2468-2481.

6. Purslow LR, Young EH, Wareham NJ, Forouhi N, Brunner EJ, Luben RN, Welch AA, Khaw KT, Bingham SA, Sandhu MS. Socioeconomic position and risk of short-term weight gain: prospective study of 14,619 middle-aged men and women. BMC Public Health. 2008; 8: 112.

7. Rosvall M, Chaix B, Lynch J, Lindström M, Merlo J. Similar support for three different life course socioeconomic models on predicting premature cardiovascular mortality and allcause mortality. BMC Public Health. 2006; 6: 203.
8. Zhou G, Liu X, Xu G, Liu X, Zhang R, Zhu W. The effect of socioeconomic status on three-year mortality after first-ever ischemic stroke in Nanjing, China. BMC Public Health. 2006; 6: 227.

9. Regidor E. La clasificación de la clase social de Goldthorpe: marco de referencia para la propuesta de medición de la clase social del grupo de trabajo de la SEE. Rev Esp Salud Publica 2001; 75 : 13-22.

10. Feito R. Estructura social contemporánea. Las clases sociales en los países industrializados. Madrid: Siglo XXI Editores SA, 1995.

11. Erikson R, Goldthorpe JH, Jackson M, Yaish M, Cox DR. On class differentials in educational attainment. Proc Natl Acad Sci U S A. 2005; 102: 9730-9733.

12. Domínguez-Berjón MF, Borrell C, Cano-Serral G, Esnaola S, Nolasco A, Pasarín MI, Ramis R, Saurina $\mathrm{C}$, Escolar-Pujolar A. Constructing a deprivation index based on census data in large Spanish cities(the MEDEA project) Gac Sanit. 2008; 22: 179-87.

13. Pérez MA, Moreno VM, Puerta DR, Martínez YG, Vicario IH, Ceruelo EE, de la Cámara AG. Socioeconomic factors and utilization of public family practice facilities in Madrid. Gac Sanit. 2007; 21 : 219-26.

14. Yanosky JD, Schwartz J, Suh HH. Associations between measures of socioeconomic position and chronic nitrogen dioxide exposure in Worcester, Massachusetts.J Toxicol Environ Health A. 2008; 71: 1593-02.

15. Whitley E, Gunnell D, Davey Smith G, Holly JM, Martin RM. Childhood circumstances and anthropometry: the Boyd Orr cohort. Ann Hum Biol. 2008; 35: 518-34.

16. Cesaroni G, Agabiti N, Rosati R, Forastiere F, Perucci CA. An index of socioeconomic position based on 2001 Census, Rome Epidemiol Prev. 2006; 30: 352-57.

17. Benach J, Amable M. Social classes and poverty. Gac Sanit. 2004; 18 Suppl 1:16-23.

18. Antonio Cabrera de León, $\mathbf{M}^{\mathrm{a}}$ del Cristo Rodríguez Pérez, Delia Almeida González, Santiago Domínguez Coello, Armando Aguirre Jaime, Buenaventura Brito Díaz, Lina I. Pérez Méndez y el grupo CDC. Presentación de la cohorte "CDC de Canarias": objetivos, diseño y resultados preliminares. Rev Esp Salud Pública. 2008; 82: 519-34. 
19. Cabrera de León A, Rodríguez Pérez M del Cristo, Rodríguez Benjumeda LM, Anía Lafuente B, Brito Díaz B, Muros de Fuentes M, Almeida González D, Batista Medina M y Aguirre Jaime A. Sedentarismo: tiempo de ocio activo frente al porcentaje del gasto energético. Rev Esp Cardiol 2007; 60: 244-250.

20. Pitanga FJ, Lessa I. Waist-to-height ratio as a coronary risk predictor among adults. Rev Assoc Med Bras. 2006; 52: 157-61.

21. Third Report of the National Cholesterol Education Program (NCEP) Expert Panel on Detection, Evaluation, and Treatment of High Blood Cholesterol in Adults (Adult Treatment Panel III) Final Report. Circulation 2002; 106: 3188-3190.

22. The IDF consensus worldwide definition of the metabolic syndrome. Available at: http://www.idf. org/home/index.cfm?node $=1429$. Última consulta, 25 de septiembre de 2008.

23. The Expert Comittee on the Diagnosis and Classification of Diabetes Mellitus: Report of the Expert Committee on the Diagnosis and Classification of Diabetes Mellitus. Diabetes Care. 1997; 20: 118397.

24. Clasificación Nacional de Ocupaciones. Instituto Nacional de Estadística. Madrid,1994.

25. Grupo de trabajo de la Sociedad Española de Epidemiología y de la Sociedad Española de Medicina Familiar y Comunitaria. Una Propuesta de medida de la clase social. Aten Primaria 2000; 25: 350-63.

26. Regidor E, Gutiérrez-Fisac JL, Banegas JR, Vicente D, Rodríguez-Artalejo F. Life socioeconomic circumstances, physical inactivity and obesity influences on metabolic syndrome. Rev Esp Salud Publica. 2007; 81: 25-31.

27. Santos AC, Ebrahim S, Barros H. Gender, socioeconomic status and metabolic syndrome in middle-aged and old adults. BMC Public Health. 2008 Feb 18; 8: 62 .

28. Marquezine GF, Oliveira CM, Pereira AC, Krieger JE, Mill JG. Metabolic syndrome determinants in an urban population from Brazil: Social class and gender-specific interaction. Int J Cardiol. 2008; 129: 259-65.

29. Sampson RJ, Sharkey P. Neighborhood selection and the social reproduction of concentrated racial inequality. Demography. 2008; 45: 1-29.

30. Shishehbor MH, Gordon-Larsen P, Kiefe CI, Litaker D. Association of neighborhood socioecono- mic status with physical fitness in healthy young adults: the Coronary Artery Risk Development in Young Adults (CARDIA) study. Am Heart J. 2008; 155: 699-705.

31. Galobardes B, Morabia A, Bernstein MS. Diet and socioeconomic position: does the use of different indicators matter? Int J Epidemiol. 2001; 30: 334340 .

32. González CA, Argilaga S, Agudo A, Amiano P, Barricarte A, Beguiristain JM, Chirlaque MD, Dorronsoro M, Martinez C, Navarro C, Quirós JR, Rodriguez M, Tormo MJ. Sociodemographic differences in adherence to the Mediterranean dietary pattern in Spanish populations. Gac Sanit. 2002; 16: 214-221.

33. Tingay RS, Tan CJ, Tan NC, Tang S, Teoh PF, Wong R, Gulliford MC. Food insecurity and low income in an English inner city. J Public Health Med. 2003; 25: 156-159.

34. Oehlschlaeger MH, Pinheiro RT, Horta B, Gelatti C, San,Tana P. Prevalence of sedentarism and its associated factors among urban adolescents. Rev Saude Publica. 2004; 38: 157-163.

35. Pikhart H, Bobak M, Malyutina S, Pajak A, Kubínová $\mathrm{R}$, Marmot M. Obesity and education in three countries of the Central and Eastern Europe: the HAPIEE study. Cent Eur J Public Health. 2007; 15: 140-142.

36. Perel P, Langenberg C, Ferrie J, Moser K, Brunner E, Marmot M. Household wealth and the metabolic syndrome in the Whitehall II study. Diabetes Care. 2006; 29: 2694-2700.

37. Agardh EE, Ahlbom A, Andersson T, Efendic S, Grill V, Hallqvist J, Ostenson CG. Socio-economic position at three points in life in association with type 2 diabetes and impaired glucose tolerance in middle-aged Swedish men and women. Int J Epidemiol. 2007; 36: 84-92.

38. Sánchez C. La clase social: concepto y medición. Dimensión Humana 1997; 1: 31-36.

39. Davis P, McLeod K, Ransom M, Ongley P, Pearce N, Howden-Chapman P. The New Zealand Socioeconomic Index: developing and validating an occupationally-derived indicator of socio-economic status. Aust N Z J Public Health. 1999; 23: 27-33.

40. Nebot M, Díez E, Benaque A, Borrel C. Maternity in adolescents in the districts and neighborhoods of Barcelona: its association with the socioeconomic level and the prevalence of low weight at birth. Aten Primaria. 1997; 19: 449-454. 
41. Díaz de Quijano E, Brugal MT, Pasarín MI, Galdós-Tanguiís $\mathrm{H}$, Caylà $\mathrm{J}$, Borrell $\mathrm{C}$. Influence of social inequality, social unrest and extreme poverty on tuberculosis morbidity in the City of Barcelona. Rev Esp Salud Publica. 2001; 75: 517-527.

42. Merchant AT, Dehghan M, Behnke-Cook D, Anand SS. Diet, physical activity, and adiposity in children in poor and rich neighbourhoods: a crosssectional comparison. Nutr J. 2007; 6: 1-7.

43. Serra-Majem LI. Encuesta Nutricional de Canarias (1997-1998). Servicio Canario de Salud. Consejería de Sanidad y Asuntos Sociales. Gobierno de Canarias. Las Palmas de Gran Canaria, 2000. Disponible en: www.gobiernodecanarias.org/ sanidad/scs/1/plansalud/enca [última consulta, 25 de septiembre de 2008].
44. Borges Álamo C. Análisis de la dieta de la población adulta de Canarias y su relación con los patrones dietéticos mediterráneo y occidental. Tesis doctoral presentada en la Universidad de La Laguna el 11 de julio de 2008. Disponible en: http://www.micinn.es/teseo. Última consulta el 25 de septiembre de 2008.

45. Daponte-Codina A, Bolivar-Muñoz J, Toro Cárdenas S, Ocaña Riola R, Benach Rovira J, NavarroLópez V. Area deprivation and trends in inequalities in self-rated health in Spain, 1987-2001. Scand J Public Health. 2008; 36: 504-515.

46. Denton M, Prus S, Walters V. Gender differences in health: a Canadian study of psychosocial, structural and behavioural determinants of health. Soc Sci Med. 2004; 58: 2585-2600. 